\title{
Sensitivity of PET/MRI to detect recurrence of prostate cancer
}

\author{
Patricia Arce-Calisaya • Michael Souvatzoglou • \\ Mathias Eiber • Ambros Beer • Klemens Scheidhauer • \\ Hans Geinitz • Markus Schwaiger
}

Received: 29 August 2012 / Accepted: 21 January 2013 /Published online: 22 February 2013

(C) Springer-Verlag Berlin Heidelberg 2013

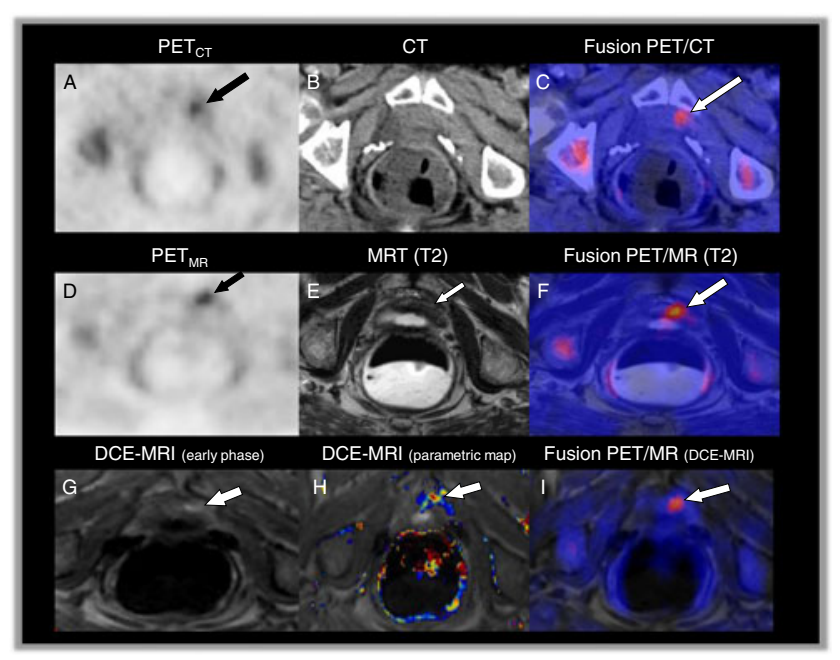

A 73-year-old gentleman underwent radical prostatectomy due to prostate cancer (2003); during his follow-up he presented with biochemical recurrence (prostate-specific antigen $2 \mathrm{ng} / \mathrm{ml}$ ) [1]. Positron emission tomography (PET)/CT with $\left[{ }^{11} \mathrm{C}\right]$ choline was performed $5 \mathrm{~min}$ after injection of $706 \mathrm{MBq}$ of $\left[{ }^{11} \mathrm{C}\right]$ choline. The images revealed a moderate uptake of the left prostate bed which had no morphologic correlate in the diagnostic CT. The configuration of the uptake and its moderate intensity (maximum standardized uptake value 2.7) made the lesion hard to be differentiated from any other unspecific finding. Hence, he underwent simultaneous whole-body PET/MRI (Siemens Biograph mMR) acquisition immediately after PET/CT. In the dynamic MRI an early contrast-enhanced lesion was found in the region of the moderate uptake seen in PET/CT. This additional finding increased the level of suspicion of malignancy.

\section{References}

1. Jadvar H. Prostate cancer: PET with $18 \mathrm{~F}-\mathrm{FDG}, 18 \mathrm{~F}$ - or $11 \mathrm{C}$-acetate, and 18F- or 11C-choline. J Nucl Med 2011;52(1):81-9.

P. Arce-Calisaya $(\bowtie)$

Nuclear Medicine Department, University Hospital

of Santiago de Compostela, Santiago, Spain

e-mail: Patricia.Arce-Calisaya@leedsth.nhs.uk

M. Souvatzoglou $\cdot$ M. Eiber $\cdot$ A. Beer $\cdot$ K. Scheidhauer $\cdot$

M. Schwaiger

Nuclear Medicine Department, Klinikum rechts der Isar,

Munich, Germany

H. Geinitz

Radiation Therapy/Radiation Oncology, Klinikum rechts der Isar,

Munich, Germany 